\title{
Time serial analysis of the induced LEO environment within the ISS 6A
}

\author{
J. W. Wilson ${ }^{\mathrm{a}}$, J. E. Nealy ${ }^{\mathrm{b}}$, Ts.P. Dachev ${ }^{\mathrm{c}}$, B.T. Tomov ${ }^{\mathrm{c}}$, F. A. Cucinotta ${ }^{\mathrm{d}}$, F. F. Badavi ${ }^{\mathrm{e}}$, G. De Angelis ${ }^{\mathrm{b}, \mathrm{f}}$, \\ W. Atwell ${ }^{\mathrm{g}}$, N. Leutke \\ ${ }^{\mathrm{a}}$ NASA Langley Research Center, Hampton, VA USA, ${ }^{\mathrm{b}}$ Old Dominion University, Norfolk, VA USA, ${ }^{\mathrm{c} B u l g a r i a n}$ \\ Academy of Science, Sofia, Bulgaria, ${ }^{\mathrm{d}}$ NASA Johnson Space Center, Houston, TX USA, ${ }^{\mathrm{C}}$ Christopher Newport \\ University, Newport News, VA USA, ${ }^{\mathrm{f}}$ Istituto Superiore di Sanità, Rome, Italy, ${ }^{\mathrm{g}}$ The Boeing Company, Houston, \\ TX USA, ' $\mathrm{S} w a$ ales Aerospace, Hampton, VA USA \\ john.w.wilson@nasa.gov/ Fax: +1-757-864-8094
}

\begin{abstract}
Anisotropies in the low Earth orbit (LEO) radiation environment were found to influence the thermoluminescence detectors (TLD) dose within the (International Space Station) ISS 7A Service Module. Subsequently, anisotropic environmental models with improved dynamic time extrapolation have been developed including westward and northern drifts using AP8 Min \& Max as estimates of the historic spatial distribution of trapped protons in the 1965 and 1970 era, respectively. In addition, a directional dependent geomagnetic cutoff model was derived for geomagnetic field configurations from the 1945 to 2020 time frame. A dynamic neutron albedo model based on our atmospheric radiation studies has likewise been required to explain LEO neutron measurements. The simultaneous measurements of dose and dose rate using four Liulin instruments at various locations in the US LAB and Node 1 has experimentally demonstrated anisotropic effects in ISS 6A and are used herein to evaluate the adequacy of these revised environmental models.
\end{abstract}

\section{INTRODUCTION}

The commitment of astronauts to long-term exposure in the space environment during future space exploration requires resolution of issues concerning ionizing radiation. For example, evidence of early cataract formation in Shuttle operations at especially high orbital inclination with its greater portion of Galactic Cosmic Rays (GCR) exposure provides added emphasis (Cucinotta et al. 2001) to the need for careful design evaluation for operations beyond the confines of the Earth's protective magnetic field. For the high inclination of the ISS $\left(51.6^{\circ}\right)$, computational models indicate that about half of the ionizing radiation exposure near solar minimum results from GCR and the bulk of the remainder from trapped particles. There is, of course, a contribution from the neutron albedo of 25 to $54 \mu \mathrm{Sv} / \mathrm{d}$ (varies with solar cycle) excluding effects of intervening material (Wilson et al. 1989). Within the spacecraft, the environment is a complex mixture of surviving primary particles and secondary radiations produced in the spacecraft structure. Various arrangements of detectors have been used to study the composition of the internal radiation fields within the spacecraft, which need to be understood in terms of computational models to allow a better understanding of the local environment at the location of the astronauts' critical tissues. As a result, a number of studies of the low Earth orbit (LEO) environment have been made to better understand the nature of the radiations within a spacecraft (Badhwar et al. 1995a, Dudkin et al. 1992, 1995, Keith et al. 1992, Lyagushin et al. 1998, Dachev et al. 2002) and to understand these results in terms of computational models (Badhwar 1995b, 2002, Shinn et al. 1995, 1998, Wilson et al. 2002, Hugger et al. 2003, Getselev et al. 2004, Nealy et al. 2007). With improved understanding of the LEO environment comes the possibility of testing new designs for future exploration systems in LEO orbital flight prior to commitment to human operations on the moon and Mars. 
In prior reports (Wilson et al. 2002, 2006a), we used a relatively complete dynamic model based on simple scaling relations of the LEO environment as related to solar activity cycle for the omni-directional flux of particles from GCR, trapped particles, and neutron albedo including directional dependence (on an ad hoc basis). In the present report, we emphasize the spatial dependence of the environment related to passage through the South Atlantic Anomaly (SAA) while a related paper (Nealy et al. 2007) considers the time averaged environment relative to the measurements of Dachev et al. (2002).

\section{ENVIRONMENTAL MODEL DEVELOPMENT}

The two primary limitations in the environmental models AP8 MIN and MAX is the assumption that the trapped particles are represented by an omni-directional description and known at the fixed epochs of 1965 and 1970 (Wilson et al. 2002, 2006a). Directionality was introduced using the work of Heckman and Nakano (1963) but without a major reformulation of the dynamics of the inner zone radiation the spatial distribution of the particles are those given by the basic historic AP8 models. The traditional geomagnetic cutoff representation was fixed in time at 1985 and used the orbital averaged vertical geomagnetic cutoff to describe the transmitted galactic cosmic rays limiting directional information and provided underestimates at low rigidity transmission and overestimates at higher rigidity. These models have been relatively successful in describing the radiation environment aboard the highly maneuverable Shuttle wherein anisotropies tend to be averaged. Such models will not be adequate in the formation flying of the ISS mainly oriented in the local horizontal plane along the velocity vector (Hugger et al. 2003). We developed new dynamic/anisotropic trapped proton environmental and general geomagnetic cutoff models (Wilson et al. 2006a, Nealy et al. 2007) to correct some of these deficiencies. In the present paper, we reexamine the spatial dependence and drift of the SAA environmental model as defined by the AP8 models. These models are placed in a suitable form for evaluation of the incident radiation on the bounding surface of the sixdegree of freedom motion of an orbiting spacecraft for evaluation of the environment within the ISS 6A model simulation in the present study.

The trapped proton population is modeled as AP8 for solar minimum (1965) and maximum (1970) conditions (Vette 1991). These inner zone particles result from the decay of atmospheric neutrons as they leak from the Earth's atmosphere into the trapping region. The inner zone particles are lost from the trapping region by interaction with the tenuous upper atmosphere and generally have long trapping lifetimes. The inner zone consists of both proton and electron decay products. The average kinetic energy of the inner zone electrons is a few hundred $\mathrm{keV}$. These electrons are easily removed from the spacecraft interior by the slightest amount of shielding and are mainly of concern to an astronaut in a spacesuit and external materials. Within any pressure vessel such as the Shuttle or ISS, the electrons are easily shielded by the micrometeoroid/debris bumper and pressure vessel wall. Of the trapped particles, only the protons with energies near or above one hundred $\mathrm{MeV}$ are of concern to the interior environment of the Shuttle or the ISS.

The particles trapped in the geomagnetic field were modeled from data obtained during two epochs of solar cycle 20 (solar minimum of 1965 and solar maximum of 1970) and are used with the geomagnetic fields on which the $\mathrm{B} / \mathrm{L}$ maps were prepared (McCormack 1988). The 1965 analysis using the magnetic field model Jensen and Cain (1962) resulted in particle population maps AP8 MIN (Sawyer and Vette 1976). The 1970 analysis using the magnetic field model GSFC 12/66 (Cain et al. 1967, extended to 1970) resulted in the particle population maps of AP8 MAX (Sawyer and Vette 1976). These models are considered the best available global representations of the trapped proton environment. This includes the known uncertainties in the AP8 models of a factor of 2 in LEO applications. The trapped protons present at $400 \mathrm{~km}$ are mainly located over the South Atlantic due to the displacement of the geomagnetic dipole moment relative to the Earth's rotational axis dragging the

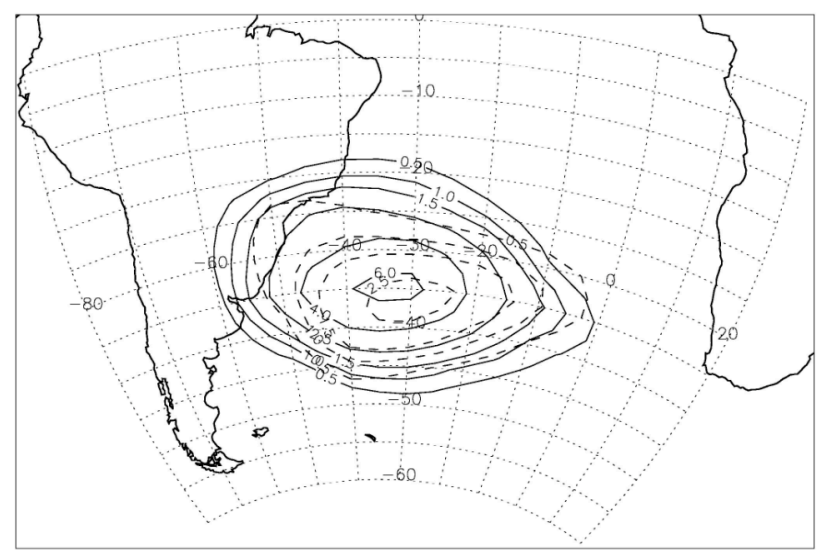

Fig. 1. Omni-directional proton flux map $(\mathrm{E}>100 \mathrm{MeV})$ at 400 $\mathrm{km}$ according to AP8 Min (solid) and Max (dashed).

Table 1. The AP8 SAA Central Locations for $100 \mathrm{MeV}$ Protons at $400 \mathrm{~km}$ During Epochs 1965 and 1970.

\begin{tabular}{|l|c|c|}
\hline SAA Location & Virgin model & Adjusted \\
\hline 1965 & & \\
Latitude & $35.0^{\circ} \mathrm{S}$ & $32.6^{\circ} \mathrm{S}$ \\
Longitude & $33.0^{\circ} \mathrm{W}$ & $37.1^{\circ} \mathrm{W}$ \\
\hline 1970 & & \\
Latitude & $37.0^{\circ} \mathrm{S}$ & $32.2^{\circ} \mathrm{S}$ \\
Longitude & $30.0^{\circ} \mathrm{W}$ & $38.0^{\circ} \mathrm{W}$ \\
\hline
\end{tabular}


trapped belts deeper into the atmosphere in this region as shown in Fig. 1. The central locations of these fields differ as a result of the five-year secular variation of the geomagnetic field but also due to the differing fidelity of the geomagnetic model used in the two epochs. For the purpose of interpolating between the two models, we have realigned the models as noted in Table 1.

It was believed at one time that better estimates of the particle environments could be gained by evaluating the population maps defined on invariant Mcllwain coordinates using current geomagnetic field conditions. This interpolation would, for example, contain the westward drift ( 0.3 degrees/yr) of the South Atlantic Anomaly (SAA) observed in recent years by Badhwar et al. (1996). However, it was recognized by the Shuttle dosimetry group (Atwell et al. 1989) that large errors resulted from such a procedure which does not account accurately for the altitude shifts with changing fields (mainly from the decline of the geomagnetic dipole moment), and it was concluded that the use of the particle population maps interpolated over the historic geomagnetic field model for which the population map was derived would provide the best estimates of the long-term orbital averaged particle environments even though the westward drift is not

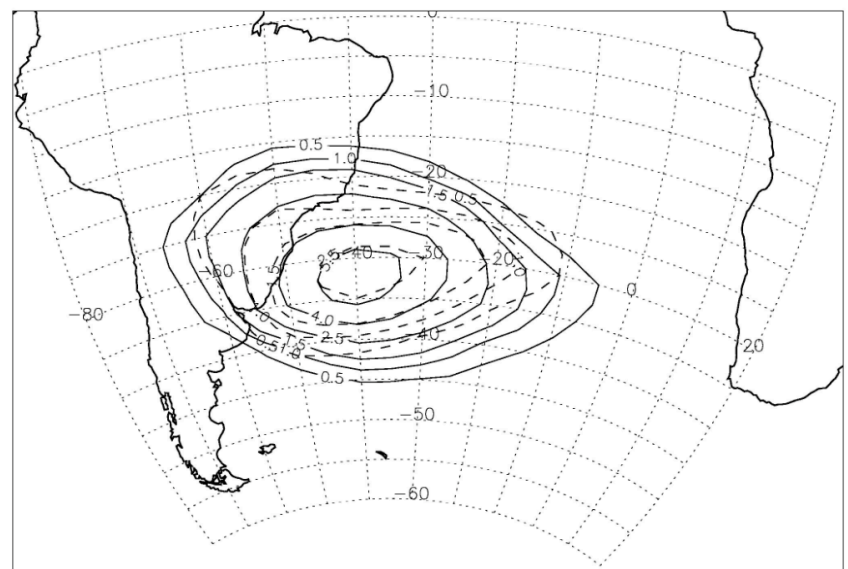

Fig. 2 Adjusted omni-directional flux map (E > $100 \mathrm{MeV})$ locations for 1970 of the South Atlantic Anomaly for AP8MIN (1965) and AP8MAX (1970). represented. The westward drift is often introduced as an eastward rotation of spacecraft geographic coordinates (0.3 degree/yr) without regard to modifying the geomagnetic field over which the AP8 models were derived (Heynderickx 1996). Note, the long-term orbital averaged environments do not depend on the westward drift.

\begin{tabular}{|l|l|l|c|c|}
\hline \multicolumn{5}{|l|}{ Table 2. The Historic SAA Central Locations $\left(\theta_{c}, \phi_{c}\right)$ from Various Sources at a Nominal $400 \mathrm{~km}}$. \\
\hline Source & Date $(\mathrm{s})$ & Quantity & Latitude, S & Longitude, $\mathrm{W}$ \\
\hline AP8 & 1970 & $50 \mathrm{MeV}$ protons & 34 & 34.2 \\
\hline & & $100 \mathrm{MeV}$ protons & 34 & 36.6 \\
\hline Skylab & $12 / 1973$ & Dose rate & 32 & 38.6 \\
\hline REM & $11 / 1994-11 / 1996$ & $50 \mathrm{MeV}$ protons & 33 & 42.1 \\
\hline & & $200 \mathrm{MeV}$ protons & 32 & 44.3 \\
\hline Mir-1 & TEPC dose rate & NA & 44.9 \\
\hline STS 101 & $3 / 1995$ & TEPC dose rate & $34 \pm 2.15$ & $48 \pm 1.8$ \\
\hline ISS & $5 / 2000$ & Liulin 130 MeV protons & 28 & 44 \\
\hline & $6-7 / 2001$ & Liulin dose rate & 29.6 & 44.2 \\
\hline Foton M2 & & $180 \mathrm{MeV}$ protons & 33 & 49.7 \\
\hline
\end{tabular}

The trapped proton environment has as its source the atmospheric neutron albedo, and the losses of trapped protons occur through atmospheric interaction (mainly energy loss but also scattering in atomic collisions). The proton environment is then proportional, in steady state, to the source (neutron albedo) and the population lifetime (atmospheric interaction) as shown by Blanchard and Hess (1964). The interpolation procedure we will implement assumes a steady state solution to the population kinetic equations as the product of the albedo neutron source and the particle population lifetimes that are proportional to the product of neutron monitor count rate and solar radio output at the $10.7 \mathrm{~cm}$ wavelength related to atmospheric heating resulting in expansion. The interpolation of the AP8 models then involves two operations. The first operation is the westward drift followed by the solar modulation through the neutron decay source and loss terms related to atmospheric interaction. To implement such a scheme, we must assure the AP8 MIN and MAX models are appropriately aligned in latitude and longitude to facilitate interpolation/extrapolation of the fixed epochs. This alignment process is given in Table 1 and shown in Fig. 2 as adjustments to the centers of the two environmental models.

The AP8 MIN and MAX models associated with epochs 1965 and 1970 use different geomagnetic field models resulting in artifacts in the data analysis in deriving the models that we will in part correct. The AP8 MIN and MAX central locations of the South Atlantic Anomaly (SAA) are given in Table 1. A historical review of the SAA central location was given by Atwell (2002) with a summary of his findings in Table 2. We assume the central location after 1970 is given by the modeled geographic locations as

$$
\begin{aligned}
& \theta_{c}=-32.2+0.07(t-1970) \\
& \phi_{c}=-38-0.19(t-1970)
\end{aligned}
$$


The modeled locations are compared to the data collected by Atwell (2002) in Figs. 3 and 4. In the present model modifications, we adjust the location of the AP8 models as given by Table 1 and drift according to equations (1) and (2) beyond 1970. In addition to the adjustment and drift, the AP8 models will be scaled and modulated according to LEO measurements in hopes to give essential modifications leading to a model that more accurately represents the current fields. Thus, $f_{A P B M A X}(r, \theta-4.8, \phi+8, E)$ as derived from AP8 MAX is adjusted by $4.8^{\circ} \mathrm{N}$ and $8^{\circ} \mathrm{W}$ while

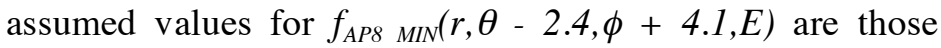
from AP8 MIN adjusted by $2.4^{\circ} \mathrm{N}$ and $4.1^{\circ} \mathrm{W}$. Using the drift equations (1) and (2), aligns the modeled proton fields in 1970 as shown in Fig. 2. A first approximation to successive solar maximum and solar minimum is found by simply allowing a northward and westward drift in addition to the above adjustments.

The proton omni-directional flux spectrum at any location and time $f_{p}(r, \theta, \phi, E, t)$ is then extrapolated (Wilson et al. 2002) using the following functional form

$$
\begin{aligned}
& f_{p}(r, \theta, \phi, E, t)=f_{p, \min }(r, \theta, \phi, E) \\
& \left.\quad \times \exp \left\{-\alpha_{p}\left[D R N M \times F_{10.7}-D R N M \times F_{10.7}\right)_{M I N}\right]\right\}
\end{aligned}
$$

where

$$
\begin{aligned}
& f_{p, \min }(r, \theta, \phi, E)=0.5 \\
& \quad \times f_{\text {AP8MIN }}(r, \theta-2.4-0.07 \Delta t, \phi+4.1+0.19 \Delta t, E)
\end{aligned}
$$

is the proton flux at solar minimum shifted to time $t$ after 1965 and $\alpha_{p}$ is evaluated using the solar maximum

$$
\begin{aligned}
& f_{p, \max }(r, \theta, \phi, E)=0.6 \\
& \quad \times f_{A P 8 M A X}(r, \theta-4.8-0.07 \Delta t, \phi+8+0.19 \Delta t, E)
\end{aligned}
$$

is the proton flux at solar maximum shifted to $t$ after 1970 and related to the AP8 MIN and MAX models but with the latitude shifted north by $0.07^{\circ} \mathrm{N} / \mathrm{yr}$ and west by $0.19^{\circ} \mathrm{W} / \mathrm{yr}$ past the adjusted coordinates of the appropriate epochs as discussed above. In equation (3), the quantity $\left(D R N M \times F_{10.7}\right)$ is averaged over the prior 14 months at solar minimum and 2 months at solar maximum with linear interpolation as determined by a best fit to the limited NOAAPRO model (Huston and Pfitzer 1998, Wilson et al. 2006a). Note the scale factors of the prior model (Wilson et al. 2006a) have decreased. The sunspot numbers and Deep River neutron monitor over current solar cycle 23 (observed and projected) are used (Kim et al. 2006).

The implementation of angular incidence is as before (Wilson et al. 2006a) and further discussed by Nealy et al. (2007). Angular effects within the current model are shown in Fig. 5 on the directional dose sphere evaluated within a US Habitat module model simulation. On the spheres in Fig. 5, one can clearly see the Earth shadow effects on the GCR arrival as the black region on the bottom of the sphere. The square figure on the front of the sphere results from the low shielding on the space end of the hallway. The diagonal slash is due to the arrival of protons

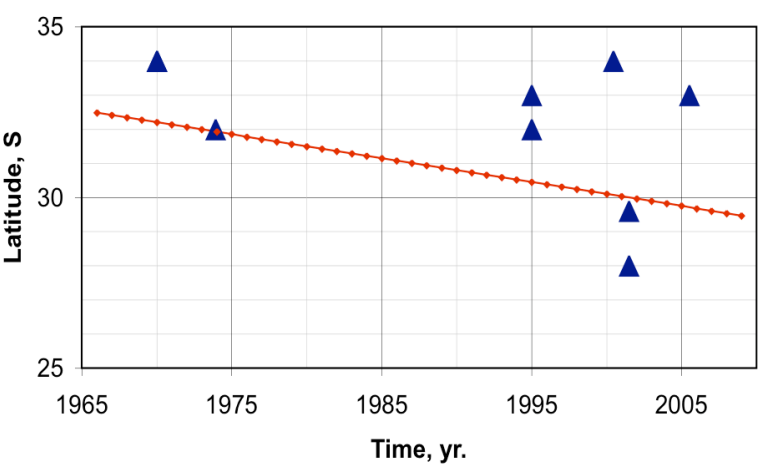

Fig. 3. Latitude drift of the current model compared with data collected by Atwell (2002).

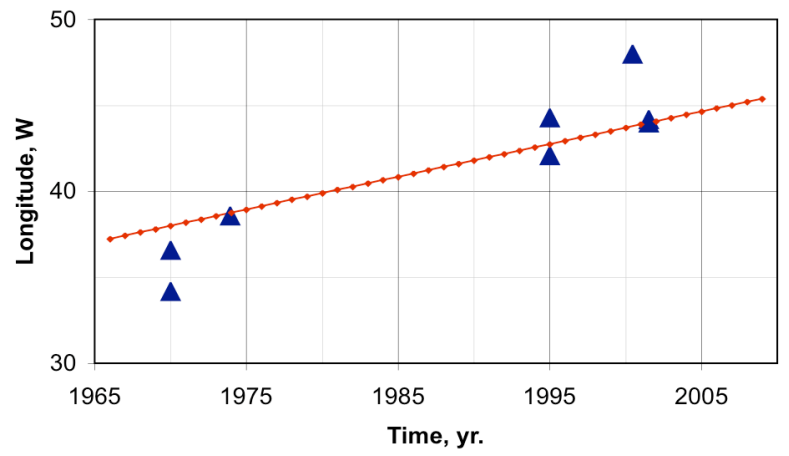

Fig. 4. Longitudinal drift of the current model compared with data collected by Atwell (2002).

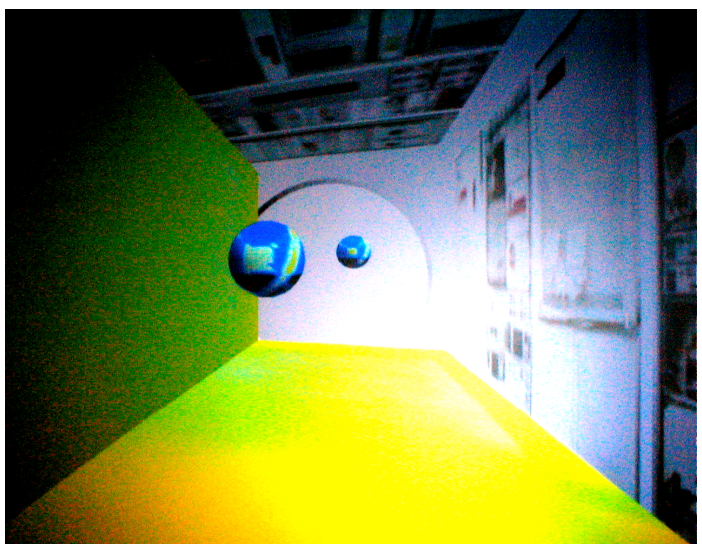

Fig. 5 Directional dose distribution predicted for ISS in passing through South Atlantic Anomaly.

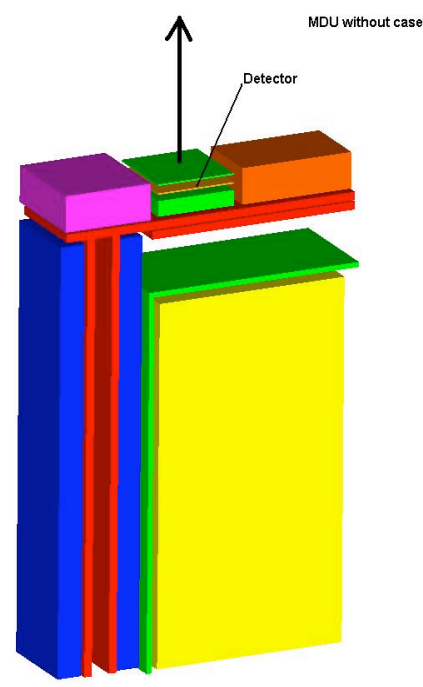

Fig. 6. MDU modeled internal parts. 
in passing through the South Atlantic Anomaly (SAA). Clearly, these features will have a strong influence on how the ISS shielding affects the interior environment and most important in comparisons with experimental measurements. The GCR and neutron environment are modeled as discussed by Wilson et al. (2006a).

\section{Experimental Description}

There are four Liulin type instruments used in the present study referred to as Mobile Dosimetry Unit 1 through 4 (MDU\#1-4). The basic silicon detector is a $16 \times 16 \mathrm{~mm}^{2}$ square that is $0.3 \mathrm{~mm}$ thick (Dachev et al. 2002). It is mounted with basic electronics and lithium ion battery for power as modeled in Fig. 6 for shielding analysis. In addition, the instrument is modeled with a $1 \mathrm{~mm}$ aluminum cover (not shown in the figure). The arrow, shown at the outward face of the detector, is used in the simulations to assure that proper orientations of the modeled instruments are preserved in the simulations. The full instrument is modeled in the simulations as shown in Fig. 6 but with the covers in place.

Each instrument analyzes energy-loss events in the detector during a 30-second time period and dumps the resulting time marked spectrum into a memory device. This device allows evaluation of fluence, dose, and energy-loss spectra for each 30-second period. This sampling rate allows approximately 180 samples of the environment for each orbital period resulting in a high-resolution spatial sample of each orbit. Although the fluence spectra are often used to approximate linear energy transfer (LET) spectra, these spectra are only approximate since the oblique angle of incidence obscures the LET associated with a given particle type and energy.

The experiments were performed on the ISS $6 \mathrm{~A}$ over the time period 11 May-26 July 2001 as part of a larger dosimetric study to define the dose distribution within the ISS. The ISS altitude and orientation were variable during this period as seen in Fig. 7, and we will utilize data from the +XVV (x-axis aligned with the velocity vector of local vertical frame; null yaw, pitch, and roll with heading in the orbital plane) between the dates of 18 June-16 July 2001 for the present study. We ignore the small limit cycle motion of the ISS during this time period. The locations of MDU\#1-4 within the US Lab Module and Node 1 are as shown in Fig. 8. A more limited preliminary study is first undertaken before a more detailed study is warranted. If sufficient agreement is reached then the more detailed study will be executed. Measurements made by MDU\#1 located in the distal end of the US Lab module as shown in Fig. 9 are used in this preliminary study. The preliminary evaluation using data measured by MDU\#1 of the above environmental models is as follows.

\section{Preliminary Evaluation of MDU\#1}

This preliminary evaluation will use the dose rate data of MDU\#1 for the time period starting 6 July 2001 as shown in Fig. 9. We focus our attention on the SAA passages by subtracting the small GCR dose rate using time series analysis methods to estimate the GCR dose rate contributions in the SAA region. Having reliable estimates of the dose rate due to the trapped environment by subtraction allows direct comparison with the SAA model derived values.

The level of detail in the geometry model of the ISS can be surmised in Fig. 8. We assume herein that the materials consist of Al 2219 that is the dominant construction material. Hence, other materials such as the micrometeoroid augmentation

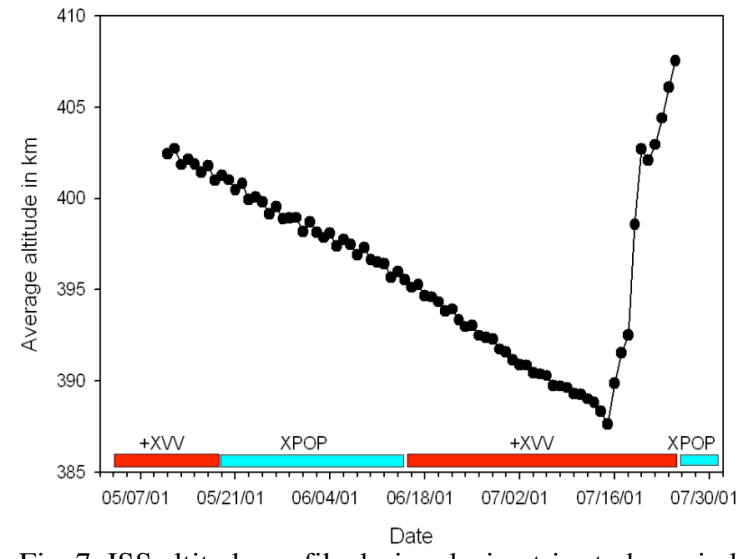

Fig. 7. ISS altitude profile during dosimetric study period.

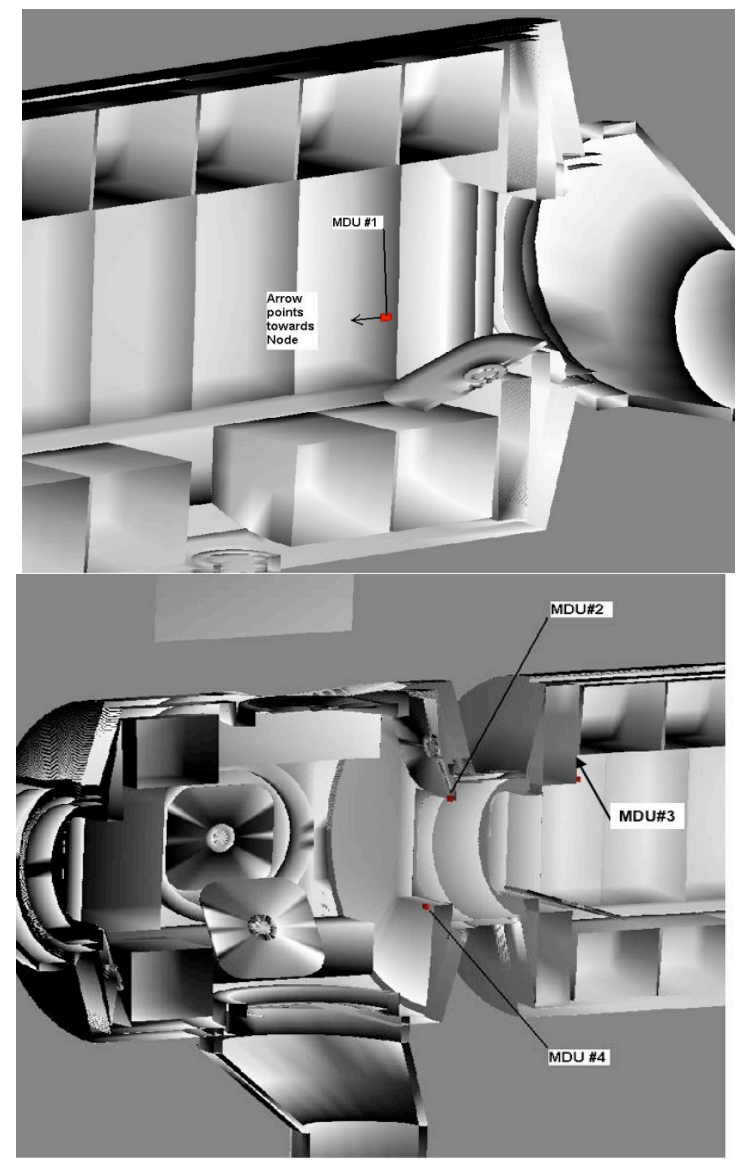

Fig. 8 MDU\# 1-4 locations in ISS 6A during the course of measurements being analyzed. Bottom figure shows Node 1 and nearest Lab locations. Top figure shows distal location of MDU\#1 in the Lab module. 
using Kevlar between the bumper and pressure vessel of those regions of ISS which are more vulnerable to debris impact are replaced by an equivalent amount of aluminum in this preliminary simulation. The boundary conditions consist of the incident fluence over each 30 -second measurement period along 970 different directions and each point on the exterior of the ISS model. In the case of the GCR we use the 30-second fraction of the average over the entire flight segment lasting 36 hours. The HZETRN 2005 code (Wilson et al. 2006b) is used to evaluate the interior environment at the location of the detector. The first analysis is based on the cumulative dose over the time period in Fig. 9 with results given in Fig. 10. In this comparison, the modeled GCR is an assumed average dose rate over the accounted time period.

The cumulative dose over the time period shows two features. First is the steady rise of dose linearly in time over extended time periods with intermittent nearly step increases. The long steady rise is the period of GCR exposure that shows very little time variation on averages over an hour or so. The near step increases arise from passages through the SAA. Note that some SAA passages are for ascending orbital nodes followed by descending nodal crossings. In these crossings, the east/west asymmetry plays a definite role as the entrance of the radiation into the ISS experiences differing amounts of shielding since heading changes have occurred from the ascending to the descending crossings. This is clearly displayed in Fig. 9 and analyzed in detail by Dachev (2006).

The measured cumulative dose is compared with the modeled dose in Fig. 10. First, one notices a growing gap in the general trends of the two results resulting from an underestimate of the GCR contribution on the order of 15 percent. This indicates a need to update the GCR model with new results by O'Neill (2006) utilizing the ACE data. Next, we see the step levels are to first order located in space and time as a result of the new drift functions used in the present calculations but that

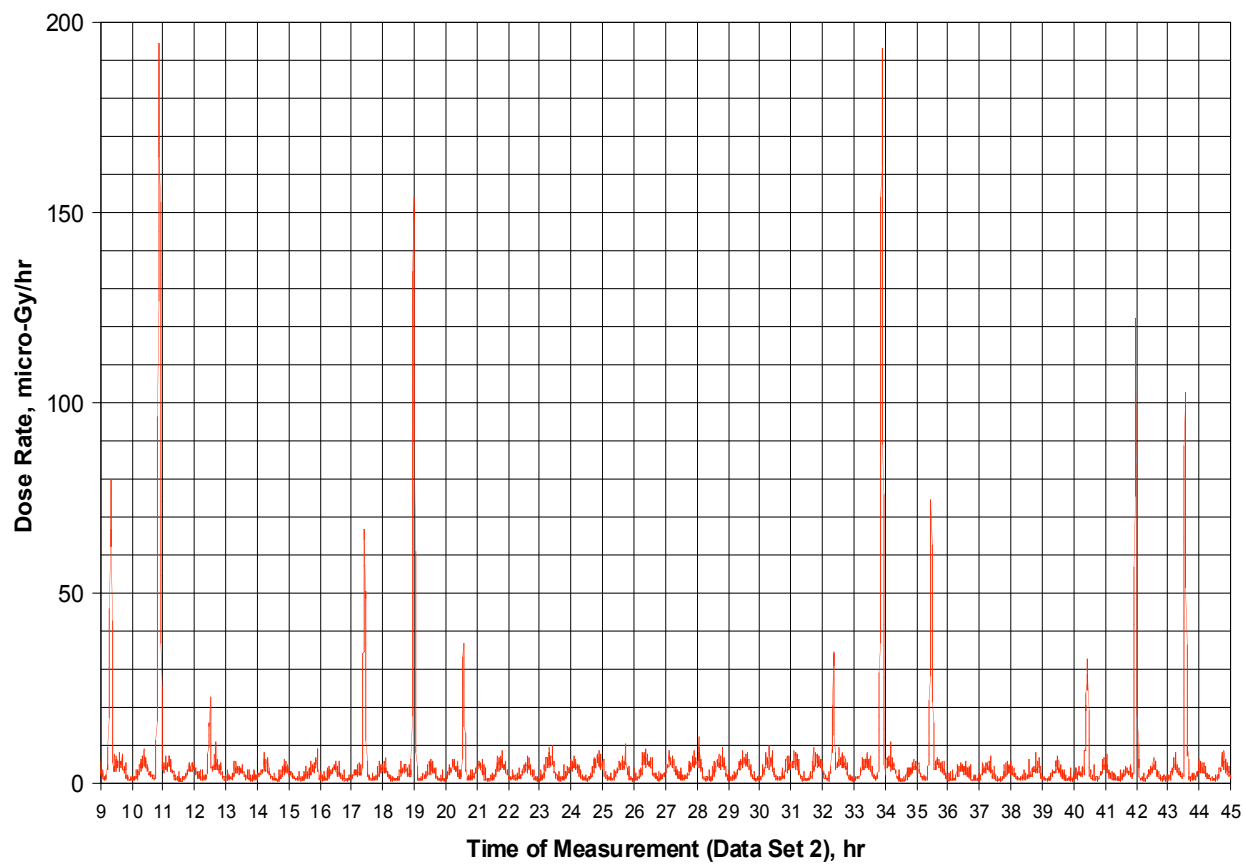

Fig. 9. MDU\#1 dose rate data starting in the $9^{\text {th }}$ hour of 6 July 2001. Large flucuations are SAA passages and small oscillations are successive passage through geomagnetic field cutoffs for galactic cosmic rays.

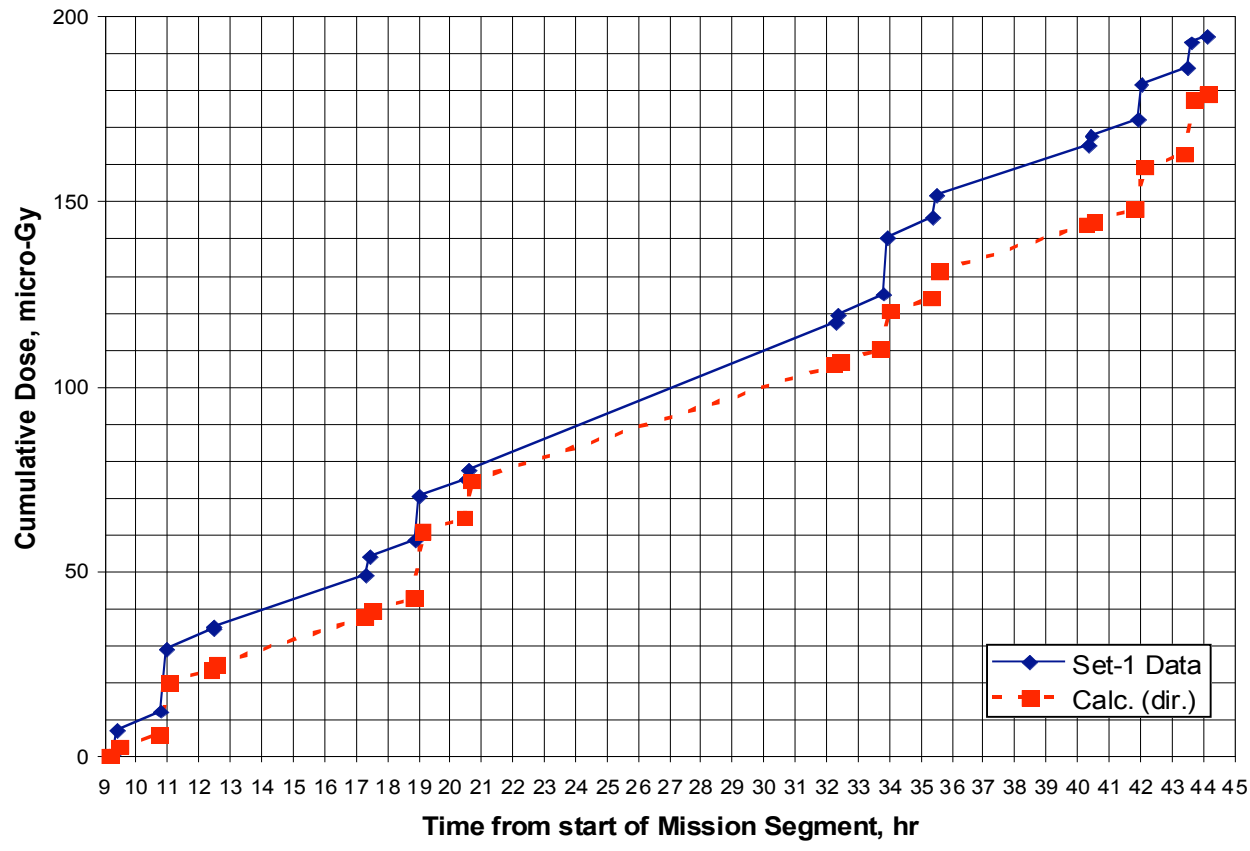

Fig. 10. Cumulative dose from MDU\#1 starting from the $9^{\text {th }}$ hour of 6 July 2001 derived from data in Fig. 9 and the computational models. 
there is some disparity on the magnitude of these increases during SAA passage. The passages are examined in greater detail by direct comparison with the 30 -second data.

We now look in detail of the step at the eleventh hour during the descending node passage in Fig. 10. Note that the step amplitude is approximately correct unlike the steps between seventeenth and twentieth hours. These differences in steps provide evidence that although the central location of the SAA is approximately correct that the spatial distribution about the center may be in error. The detailed structure of the eleventh hour step is shown in Fig. 11 with the GCR component subtracted from the experimental data as discussed above. There is an approximate 2-minute lead observed in the model values but the magnitude is approximately correct as is the cumulative dose across the passage. A dose rate contour map (normalized to unity at the maximum) was constructed from all of the measurements of the Liulin system and shown in Fig. 12 with that segment of the eleventh hour trajectory. The singular peak in the measured data occurs within the highest contour located well off the coast of Brazil. Note the general tilt south of the eastern edge of the central high dose rate region. The $100 \mathrm{MeV}$ proton flux map (also normalized to unity) is shown in Fig. 13 along with the eleventh hour trajectory for comparison. Overall, the modeled contours seem reasonably located with respect to the measurements but details reveal subtle differences. First the most intense model region is tilted north on the eastern edge in distinction to the Liulin data resulting in the 2-minute shift of the measured peak dose rate of Fig. 11. Secondly, the western limb of the outer contours of the Liulin data terminate to the east of Chile while modeled values extend well into the Pacific resulting in an early rise of modeled dose rates in Fig. 11. It is clear from these results that the assumption of drifting the basic AP8 models in time including basic adjustments to the fixed epoch maps prior to drift is limited by the spatial dependence of these historic maps. Clearly, an approach that is independent of the historic geomagnetic fields is necessary.

\section{A Possible Next Step}

The distribution of trapped protons is determined by the geomagnetic field distribution and interactions with the atmosphere. The basic AP8 models consist of population maps in $\mathrm{B} / \mathrm{L}$ coordinates that are influenced by the geomagnetic model used to reduce the satellite data obtained at specific

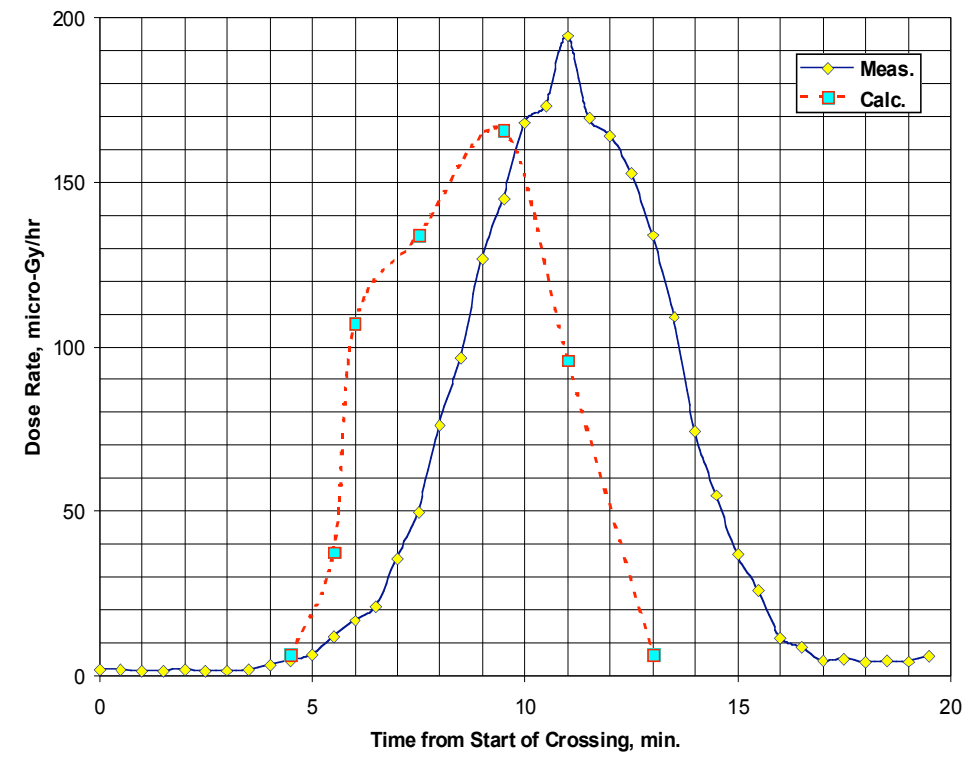

Fig. 11. Measured and calculated dose rate in a single passage through the SAA at the eleventh hour on 6 July 2001. See trajectory in Figs. 12 and 13.

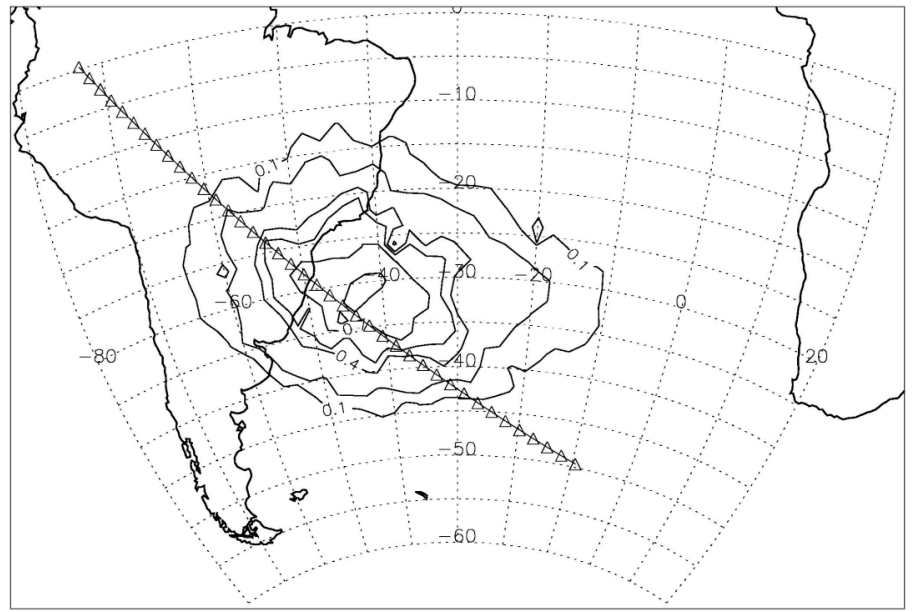

Fig. 12. ISS passage through dose rate contour map of MDU\#1-4 data sets

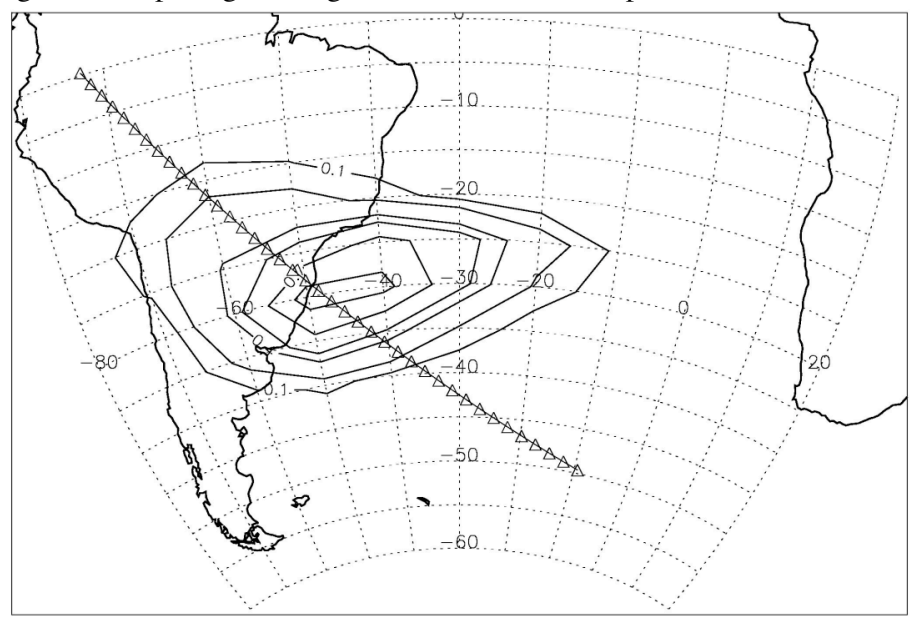

Fig. 13. ISS passage through modeled $100 \mathrm{MeV}$ proton flux contour map. 
geographic coordinates in generating the AP8 models. The atmospheric interaction appears as a rapid decline in particle populations at low $\mathrm{L}$ values and must be preserved in any model modifications.

The AP8 MAX model interpolated on the IGRF 2001 field as a contour map of protons greater than 100 $\mathrm{MeV}$ is shown in Fig. 14. No attempt has been made to make corrections to justify the AP8 MAX model to 2001 geomagnetic field conditions, especially in regard to the atmospheric cutoff. What we can see in Fig. 14 is the general westward drift reasonably following the Liulin data shown in Fig. 12. There is some hope in this procedure if the coupling to the AP8 models can be made with appropriate corrections for atmospheric interactions. One possible approach was taken in the work of Huston and Pfitzer (1998) in developing the NOAAPRO model while facing the same issue. In this case, the NOAAPRO model used maps established on a $\zeta$ and $\mathrm{L}^{\prime}$ grid where $\zeta$ is the equatorial pitch angle for the $\mathrm{B}, \mathrm{L}$ mirror point and $\mathrm{L}^{\prime}$ is the coordinate $\mathrm{L}-\mathrm{L}_{0}(\zeta)$ on the Earth's surface $(\mathrm{h}=0 \mathrm{~km})$ instead of the usual B,L grid. Note this adjustment relative to the Earth's surface allows an L' dependent map which adjusts the altitude scale relative to surface approximately correcting the map for the height of the atmosphere as required. This rescaling of the basic AP8 models will make vital adjustments in the contours in Fig. 14 and hopefully make improvements in AP8 usage over

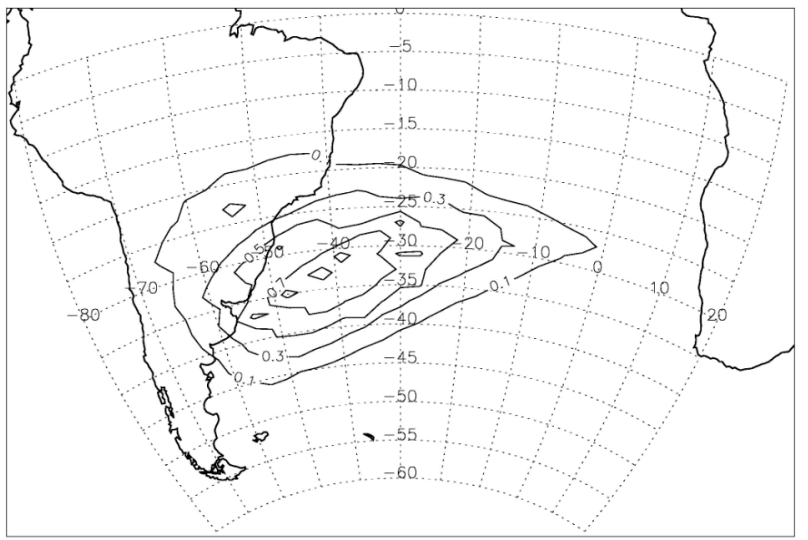

Fig. 14. Contours for protons above $100 \mathrm{MeV}$ at $400 \mathrm{~km}$ interpolated over 2001 IGRF. that of the drifting approximation used in the present work.

\section{Concluding Remarks}

Although the use of physics based dynamic modeling of the AP8 trapped radiation environment has proven remarkably accurate for orbit integrated fluence, the details of the spatial distribution are still limited by the geomagnetic field models used in generating the historic fields. Even the drifting of the fields in latitude and longitude will not allow sufficient corrections. There is some hope that developing methods for use of current geomagnetic field models with corrections of atmospheric interaction effects will result in a more accurate future model that can be applied with better convidence to projected long-term stays in LEO and validation of the future exploration shield designs.

\section{References}

Atwell, W., et al., Space radiation shielding analysis and dosimetry for the space Shuttle program. In High-Energy Radiation Background in Space, Eds. A. C. Rester \& J. I. Trombka, AIP Conference Preceedings 186, New York, 1989, pp. 289-296.

Atwell, W., A summary of the South Atlantic Anomaly (SAA) drift motion and trapped proton enhancements in the SAA. $34^{\text {th }}$ Scientific Assembly of the Committee on Space Research, Houston, Texas, October 10-19, 2002. Paper \#PSRB1-0001-02.

Badhwar G.D., et al., A study of the radiation environment on board the space Shuttle flight STS-57. Radiat. Meas. 24: 283-289; 1995a.

Badhwar, G.D., et al., Measurements of secondary particle energy spectrum in the Space Shuttle. Radiat. Meas., 24: 129-138 1995b.

Badhwar, G.D., et al., In-flight radiation measurements on STS-60. Radiat. Meas. 26:17-34; 1996.

Badhwar, G.D., et al., Space radiation absorbed dose distribution in a human phantom. Radiat. Res. 157: 76-91, 2002.

Blanchard, R.C., Hess, W.N., Solar cycle changes in inner zone protons. J. Geophys. Res. 69:3927; 1964.

Cain, J.C., et al., A proposed model for the international geomagnetic reference field-1965. J. Geomag. Geoelec. $19,335-355,1967$.

Cucinotta, F.A., et al., Space radiation and cataracts in astronauts. Radiat. Res. 156:460-466; 2001.

Dachev, Ts., et al., Calibration results obtained with Liulin-4 type dosimeters. Adv. Space Res. 30(4): 917-925; 2002. 
Dachev, Ts., Observations of the SAA radiation distribution by Liulin-E094 instrument on ISS. Adv. Space Res., 37: 1672-1677; 2006.

Dudkin, V.E., et al., Neutron fluences and energy spectra in the Cosmos-2044 Biosatellite orbit. Nucl. Tracks Radiat. Meas. 20:139-141; 1992.

Dudkin V.E., et al., Studying radiation environment on board STS-55 and STS-57 by the method of passive detectors. Radiat. Meas. 25: 483-484; 1995.

Getselev, I., et al., Absorbed dose of secondary neutrons from galactic cosmic rays inside the international space station. Adv. Space Res. 34: 1429-1432; 2004.

Heckman, H.H, Nakano, G.H., East-west asymmetry in the flux of mirroring geomagnetic trapped protons. $J$. Geophys. Res. 68: 2117-2120; 1963.

Heynderickx, D., Comparison between methods to compensate for the secular motion of the South Atlantic Anomaly. Radiat. Meas. 26: 369-373; 1996.

Huston, S.L., Pfitzer, K.A., Space environment effects: Low-altitude trapped radiation model. NASA/CR-1998208593, 1998

Hugger, C. P., et al., Preliminary validation of an ISS radiation shielding model. AIAA 2003-6220, 2003.

Jensen, D.C., Cain, J.C., An interim geomagnetic field. J. Geophys. Res. 67, 3568-3569, 1962.

Keith, J.E., Badhwar, G.D., Lindstrom, D.J., Neutron spectrum and dose-equivalent in Shuttle flights during solar maximum. Nucl. Tracks Radiat. Meas. 20: 41-47; 1992.

Kim, M. H.,Wilson, J.W., Cucinotta, F.A., A solar cycle statistical model for the projection of space radiation environment. Adv. Space Res. 37: 1741-1748; 2006.

Lyagushin, F.I., et al., Russian measurements of neutron energy spectra on the MIR orbital station. Proceedings of Workshop of Radiation and Measurements of Secondary Neutrons in Space, Houston, Texas, 1998.

McCormack, P.D., Radiation dose and shielding for the Space Station. Acta Astronaut. 17, 231-241, 1988.

Nealy, J.E., et al., Pre-engineering spaceflight validation of environmental models and the 2005 HZETRN simulation code. Adv. Space Res., in preparation (2007).

O’Neill, P.M., Badhwar-O'Neill Galactic cosmic ray model update based on advanced composition explorer (ACE) energy spectra from 1997 to present. Adv, Space Res., 37(9): 1727-1733; 2006.

Sawyer, D.M., Vette, J.I., AP-8 Trapped Proton Environments for Solar Maximum and Solar Minimum. NSSDC/WDC-A-R\&S 76-06, 1976.

Shinn, J.L., et al., Effects of target fragmentation on evaluation of LET spectra from space radiations in low Earth orbit (LEO) environment-Impact on SEU predictions. IEEE Trans. Nucl. Sci. 42: 2017-2025; 1995.

Shinn, J.L., et al., Validation of a compreshnsive space radiation transport code. IEEE Trans. Nucl. Sci. 45: 2711$2719 ; 1998$.

Vette, J.I.,The NASA/National Space Science Data Center Trapped Radiation Environmental Model Program (1964-1991). NSSDC/WDC-A-R\&91-29. 1991.

Wilson, J.W., Townsend, L.W., Farhat, H., Cosmic-ray neutron albedo dose in low-Earth orbits. Health Phys. 57: $665-668 ; 1989$.

Wilson, J.W. et al., Natural and Induced Environment in Low Earth Orbit. NASA/TM-2002-211668, 2002.

Wilson, J.W., et al., International Space Station: A test bed for experimental and computational dosimetry. Adv. Space Res. 37: 1656-1663; 2006a.

Wilson, J.W., et al., Standardized Radiation Shield Design Methods: 2005 HZETRN. SAE ICES 2006-01-2109; 2006 b. 